\title{
On the Growth Order and Growth Type of Entire Functions of Several Complex Matrices
}

\author{
M. Abul-Ez $\mathbb{D}{ }^{1}{ }^{1}$ H. Abd-Elmageed, ${ }^{2}$ M. Hidan $\left(\mathbb{D},{ }^{3}\right.$ and M. Abdalla $\mathbb{D}^{2,3}$ \\ ${ }^{1}$ Mathematics Department, Faculty of Science, Sohag University, Sohag 82524, Egypt \\ ${ }^{2}$ Mathematics Department, Faculty of Science, South Valley University, Qena 83523, Egypt \\ ${ }^{3}$ Mathematics Department, Faculty of Science, King Khalid University, Abha, Saudi Arabia \\ Correspondence should be addressed to M. Abdalla; moabdalla@kku.edu.sa
}

Received 11 June 2019; Accepted 3 September 2019; Published 17 February 2020

Academic Editor: Ismat Beg

Copyright (c) 2020 M. Abul-Ez et al. This is an open access article distributed under the Creative Commons Attribution License, which permits unrestricted use, distribution, and reproduction in any medium, provided the original work is properly cited.

In this paper, we establish an explicit relation between the growth of the maximum modulus and the Taylor coefficients of entire functions in several complex matrix variables (FSCMVs) in hyperspherical regions. The obtained formulas enable us to compute the growth order and the growth type of some higher dimensional generalizations of the exponential, trigonometric, and some special FSCMVs which are analytic in some extended hyperspherical domains. Furthermore, a result concerning linear substitution of the mode of increase of FSCMVs is given.

\section{Introduction}

The study of the asymptotic mode of increase behavior of entire functions in one and several complex variables is one of the classical central topics in complex analysis. Basic tools to study the mode of increase behavior of holomorphic functions are entities such as growth orders, the growth type, the maximum term, and the central index. Several developments made in this direction started with the early works of Lindelöf [1], Pringsheim [2], Valiron [3], Shah [4, 5], and Gol'dberg [6]. Their results turned out to be very useful in the study of partial differential equations and elsewhere. Generalization to higher dimensions has been given by many other authors (see, e.g., [7-10]) where they have contributed to the study of the order and type of entire functions of several complex variables. As Clifford analysis offers another possibility of generalizing complex function theory to higher dimensions, many authors introduced a study on the mode of increase of entire monogenic functions (see [11-16]). In recent years, the theory of matrix functions has grown significantly, with new applications appearing and the literature expanding at the last rate (see $[12,17])$. From this point of view, many authors have dealt with different problems concerning functions in several complex matrix variables (see [18-20]). However, a number of central questions concerning the asymptotic growth behavior of functions in several complex matrix variables are still open. Recently, Kishka et al. [18] obtained the order and type of entire functions of two complex matrices in complete Reinhardt domains. The present paper is devoted to investigate the mode of increase of entire functions in several complex matrix variables in hyperspherical regions independent of the scalar entire functions of several complex variables associated with them (e.g., [16, 17]). The obtained results are applicable by illustrative examples. We end our study with an interesting result on linear substitution of entire functions in several complex matrix variables in hyperspherical regions.

Following Nassif [21], we give some notations and associated properties in the framework of several complex variables.

Assume $\mathbf{m}=\left(m_{1}, m_{2}, \ldots, m_{k}\right)$ and $\mathbf{n}=\left(n_{1}, n_{2}, \ldots, n_{k}\right)$ belonging to $\mathbb{N}_{0}^{k}$ are $k$-dimensional multi-indices, and

$$
\begin{aligned}
{[\mathbf{m}] } & =m_{1}+m_{2}+\cdots+m_{k}, \\
{[\mathbf{n}] } & =n_{1}+n_{2}+\cdots+n_{k}, \\
\mathbf{z}^{\mathbf{m}} & =\left(z_{1}^{m_{1}} \cdot z_{2}^{m_{2}} \cdots \cdots z_{k}^{m_{k}}\right), \\
\mathbf{t}^{\mathbf{m}} & =\left(t_{1}^{m_{1}} \cdot t_{2}^{m_{2}} \cdots \cdots t_{k}^{m_{k}}\right), \\
0 & =(0,0, \ldots, 0),
\end{aligned}
$$


where $z_{1}, z_{2}, \ldots, z_{k} \in \mathbb{C}$ and $t_{1}, t_{2}, \ldots, t_{k}$ are nonnegative numbers. For the $k$-dimensional space, we write $\mathbf{z}=$ $\left(z_{1}, z_{2}, \ldots, z_{k}\right)$ and $\mathbf{t}=\left(t_{1}, t_{2}, \ldots, t_{k}\right)$, and for $r>0$, we set

$$
\begin{aligned}
& \mathbf{S}_{r}=\left\{\mathbf{z} \in \mathbb{C}^{k}:|\mathbf{z}|<r\right\}, \\
& \overline{\mathbf{S}}_{r}=\left\{\mathbf{z} \in \mathbb{C}^{k}:|\mathbf{z}| \leq r\right\},
\end{aligned}
$$

where $\mathbf{S}_{r}$ is an open spherical region of radius $r$ and $\overline{\mathbf{S}}_{r}$ is its closure.

Consider the function $f(\mathbf{z})$, which is regular in $\overline{\mathbf{S}}_{r}$; then, $($ see $[19,21])$

$$
f(\mathbf{z})=\sum_{[\mathbf{m}]=0}^{\infty} a_{\mathbf{m}} \mathbf{z}^{\mathbf{m}}
$$

The maximum modulus of $f(\mathbf{z})$ is denoted by

$$
M\left[f ; \overline{\mathbf{S}}_{r}\right]=\sup _{\mathbf{z} \in \overline{\mathbf{S}}_{r}}|f(\mathbf{z})| .
$$

Note that (2) leads to

$$
\left\{\left|z_{s}\right| \leq r t_{s}:|\mathbf{t}|=1 ; s=1,2, \ldots, k\right\} \subset \overline{\mathbf{S}}_{r} .
$$

Cauchy's inequality for functions of several complex variables was introduced by Nassif in the form (see [21])

$$
\left|a_{\mathbf{m}}\right| \leq \sigma_{\mathbf{m}} \frac{M\left[f ; \overline{\mathbf{S}}_{r}\right]}{r^{[\mathbf{m}]}},
$$

where

$$
\sigma_{\mathbf{m}}=\inf _{|\mathbf{t}|=1} \frac{1}{\mathbf{t}^{\mathbf{m}}}=\frac{\{[\mathbf{m}]\}^{[\mathbf{m}] / 2}}{\prod_{s=1}^{k} m_{s}^{m_{s} / 2}}
$$

and $1 \leq \sigma_{\mathbf{m}} \leq(\sqrt{k})^{[\mathbf{m}]}$ on the assumption that $m_{s}^{m_{s} / 2}=1$, whenever $m_{s}=0$.

The number $\sigma_{\mathrm{m}}$ in (7) is considered to be a generalization to the number $\sigma_{h, k}$ in the two-complex variable case (cf. [22]), where

$$
\sigma_{h, k}= \begin{cases}\frac{(h+k)^{(h+k) / 2}}{h^{h / 2} k^{k / 2}}, & h, k \geq 1, \\ 0, & h \text { or } k=0 .\end{cases}
$$

Now, the radius of convergence of the power series (3) is defined in the open sphere $\mathbf{S}_{r}$ by

$$
R_{f}=\frac{1}{\lim \sup _{[\mathbf{m}] \longrightarrow \infty}\left\{\left|a_{\mathbf{m}}\right| / \sigma_{\mathbf{m}}\right\}^{1 /[\mathbf{m}]} .}
$$

Then, the function $f(\mathbf{z})$ is an entire function if $R_{f}=\infty$. The growth order of the function $f(\mathbf{z})$ is given in [21] in the form

$$
\varrho=\limsup _{r \rightarrow \infty} \frac{\ln \ln M\left[f ; \overline{\mathbf{S}}_{r}\right]}{\ln r}=\limsup _{[\mathbf{m}] \longrightarrow \infty} \frac{[\mathbf{m}] \ln [\mathbf{m}]}{\ln \left(\sigma_{\mathbf{m}} /\left|a_{\mathbf{m}}\right|\right)} .
$$

If $0<\varrho<\infty$, we can prove using the same way as in the single complex variable case (see [23-25]) that the growth type $\theta$ of $f(\mathbf{z})$ can be given in the form

$$
\theta=\limsup _{r \rightarrow \infty} \frac{\ln M\left[f ; \overline{\mathbf{S}}_{r}\right]}{r^{\varrho}}=\frac{1}{\varrho \varrho} \limsup _{[\mathbf{m}] \longrightarrow \infty}[\mathbf{m}]\left\{\frac{\left|a_{\mathbf{m}}\right|}{\sigma_{\mathbf{m}}}\right\}^{\varrho /[\mathbf{m}]} .
$$

\section{Functions of Several Complex Matrix Variables (FSCMVs)}

We shall cite some preliminaries and notations which are essential to establish our main results (see [20]).

2.1. Preliminaries and Notations. Let $\mathscr{M}_{N}(\mathbb{C})$ be the space of $N \times N$ matrices whose entries are complex numbers. Let $A \in \mathscr{M}_{N}(\mathbb{C}), A=\left(a_{u v}\right), u, v=1,2, \ldots, N$. The multiplication law of matrices takes the form

$$
\left\{A^{2}\right\}_{u v}=\sum_{s=1}^{N}\{A\}_{u s}\{A\}_{s v},
$$

and in general,

$$
\left\{A^{\mu}\right\}_{u v}=\sum_{j_{1}, j_{2}, \ldots, j_{\mu-1}}\{A\}_{u j_{1}}\{A\}_{j_{1} j_{2}} \ldots\{A\}_{j_{\mu-2} j_{\mu-1}}\{A\}_{j_{\mu-1} v}, \quad \mu \in \mathbb{N},
$$

where the summation includes all symbols $j_{v}$ independently, from 1 to $N$. Following [20], suppose that $A, B \in \mathscr{M}_{N}(\mathbb{C}), A=\left(a_{u v}\right), B=\left(b_{u v}\right) u, v=1,2, \ldots, N$. We shall use the following notation:

$$
\{|A|\}_{u v}=\left|\{A\}_{u v}\right|,
$$

which means that a matrix $A$ whose each of its elements have been taken to be moduli of the elements.

If a matrix $B$ has positive elements which are greater than the elements of the matrix $|A|$, we have $|A|<B$. In other words, this inequality is equivalent to the following system of $N \times N$ inequalities:

$$
\left|\{A\}_{u v}\right|<\{B\}_{u v}, \quad u, v=1,2, \ldots, N .
$$

Also referring to [20], the notation $\|d\|$ shall mean that a matrix in which all its elements are equal to the number $d$ and determine its positive integral powers as follows:

$$
\left\{\|d\|^{2}\right\}_{u v}=d d+d d+\cdots+d d=N d^{2}, \quad u, v=1,2, \ldots, N .
$$

Thus, $\|d\|^{2}=\|N d\|^{2}$, and generally for positive integral powers we have

$$
\|d\|^{\mu}=\left\|N^{\mu-1} d^{\mu}\right\|, \quad \mu \in \mathbb{N} .
$$

2.2. Convergence Property of FSCMVs. In the light of Section 2.1 , we discuss convergence property of a power series of several complex matrix variables in hyperspherical regions by the convergence of a power series of several complex variables without any restrictions on the coefficients.

Let $\mathbf{X}=\left(\left[x_{s: i j}\right]\right) ; s=1,2, \ldots, k$ be commutative matrices in $\mathscr{M}_{N}(\mathbb{C})$, and then the function $\mathscr{F}(\mathbf{X})$ of several complex matrices can be written as a power series in the form 


$$
\begin{aligned}
\mathscr{F}(\mathbf{X}) & =\sum_{[\mathbf{n}]=0}^{\infty} a_{n_{1}, n_{2}, \ldots, n_{k}}, X_{1}^{n_{1}}, X_{2}^{n_{2}}, \ldots, X_{k}^{n_{k}}, \\
& =\sum_{[\mathbf{n}]=0}^{\infty} a_{\mathbf{n}} \mathbf{X}^{\mathbf{n}}=\sum_{[\mathbf{n}]=0}^{\infty} a_{\mathbf{n}} Z .
\end{aligned}
$$

Since $Z=\left[\{Z\}_{i j}\right]_{1 \leq i, j \leq N}$ and $Z \in \mathscr{M}_{N}(\mathbb{C}), Z$ may enjoy the same notation given in Section 2.1.

Thus, we write

$$
\begin{aligned}
Z= & X_{1}^{n_{1}}, X_{2}^{n_{2}}, \ldots, X_{k}^{n_{k}}, \\
\{Z\}_{i j}= & \sum_{s_{1}, s_{2}, \ldots, s_{k-1}}\left\{X_{1}^{n_{1}}\right\}_{i s_{1}},\left\{X_{2}^{n_{2}}\right\}_{s_{1} s_{2}}, \ldots,\left\{X_{k}^{n_{k}}\right\}_{s_{k-1} j} \\
= & \sum_{s_{1}, s_{2}, \ldots, s_{k-1}} \sum_{j_{1}^{(1)}, j_{2}^{(1)}, \ldots, j_{n_{1}-1}^{(1)}} x_{1 ; i j_{1}^{(1)}}, x_{1 ; j_{1}^{(1)} j_{2}^{(1)}, \ldots, x_{1 ; j_{n_{1}-1} s_{1}},} \\
& \sum_{j_{1}^{(2)}, j_{2}^{(2)}, \ldots, j_{n_{2}-1}^{(2)}} x_{2 ; s_{1} j_{1}^{(2)}, x_{2 ; j_{1}^{(2)} j_{2}^{(2)}}, \ldots, x_{2 ; j_{n_{2}-1}^{(2)} s_{2}},} \\
& \sum_{j_{1}^{(k)}, j_{2}^{(k)}, \ldots, j_{n_{k}-1}^{(k)}} x_{k ; s_{k-1} j_{1}^{(k)}} x_{k ; j_{1}^{(k)} j_{2}^{(k)} \ldots x_{k ; j_{n_{k}-1}^{(k)} j}}
\end{aligned}
$$

Hence, $\mathscr{F}(\mathbf{X})=\left[f_{i j}\right]_{1 \leq i, j \leq N}$, where

$$
f_{i j}=\sum_{[\mathbf{n}]=0} a_{\mathbf{n}}\{Z\}_{i j}, \quad i, j=1,2, \ldots, N
$$

Let us investigate the convergence of series (20) in a domain which is a subset of the space $\mathscr{M}_{N}(\mathbb{C})$ determined by the following inequalities:

$$
\left|X_{1}\right|<\left\|r t_{1}\right\|,\left|X_{2}\right|<\left\|r t_{2}\right\|, \ldots,\left|X_{k}\right|<\left\|r t_{k}\right\| .
$$

For this purpose, let

$$
\left|f_{i j}\right|=\left|\sum_{[\mathbf{n}]=0} a_{\mathbf{n}}\{Z\}_{i j}\right|, \quad i, j=1,2, \ldots, N .
$$

The convergence of this series guarantees the convergence of series (20), and in this case, series (20) will be absolutely convergent.

To show the sufficient condition for the absolute convergence of series (20), suppose that the scalar function $F(\mathbf{z})=\sum_{[\mathbf{n}]=0} a_{\mathbf{n}} \mathbf{z}^{\mathbf{n}}$ of several complex variables associated with the matrix function in (18) is an analytic function in the region $\overline{\mathbf{S}}_{N R}$, where $N R$ is the radius of convergence of this series, $N$ is the common order of our matrices, and $R$ is a positive number. Since

$$
\begin{aligned}
F(\mathbf{z}) & =\sum_{[\mathbf{n}]} a_{\mathbf{n}} \mathbf{z}^{\mathbf{n}}, \\
M\left[F ; \overline{\mathbf{S}}_{N R}\right] & =\sup _{\mathbf{z} \in \overline{\mathbf{S}}_{N R}}|F(\mathbf{z})|,
\end{aligned}
$$

using similar procedure in deriving (6), we can deduce the following inequality for the coefficients of series (23), taking into account the common order of matrices $N$. Thus,

$$
\left|a_{\mathbf{n}}\right| \leq \frac{\sigma_{\mathbf{n}} M\left[F ; \overline{\mathbf{S}}_{N R}\right]}{(N R)^{[\mathbf{n}]}},
$$

where

$$
\sigma_{\mathbf{n}}=\inf _{|\mathbf{t}|=1} \frac{1}{\mathbf{t}^{\mathbf{n}}}=\frac{\{[\mathbf{n}]\}^{[\mathbf{n}] / 2}}{\prod_{s=1}^{k} n_{s}^{n_{s} / 2}}
$$

We obviously have

$$
\begin{aligned}
\left|X_{1}\right|^{n_{1}} & <\left\|N^{n_{1}-1}\left(r t_{1}\right)^{n_{1}}\right\|,\left|X_{2}\right|^{n_{2}}<\left\|N^{n_{2}-1}\left(r t_{2}\right)^{n_{2}}\right\|, \ldots,\left|X_{k}\right|^{n_{k}} \\
& <\left\|N^{n_{k}-1}\left(r t_{k}\right)^{n_{k}}\right\| .
\end{aligned}
$$

Thus, using (24) and (26) in (22), it follows that

$$
\begin{aligned}
\left|f_{i j}\right| & =\left|\sum_{[\mathbf{n}]=0} a_{\mathbf{n}}\{Z\}_{i j}\right| \leq \sum_{\mathbf{n}}\left|a_{\mathbf{n}}\right| \sum_{s_{1}, s_{2}, \ldots, s_{k-1}}\left|\left\{X_{1}^{n_{1}}\right\}_{i_{1}}\right|, \\
& \left|\left\{X_{2}^{n_{2}}\right\}_{s_{1} s_{2}}\right|, \ldots,\left|\left\{X_{k}^{n_{k}}\right\}_{s_{k-1} j}\right| \\
& \leq \sum_{[\mathbf{n}]=0}\left|a_{\mathbf{n}}\right| N^{n_{1}-1+n_{2}-1+\cdots+n_{k}-1+(k-1)} r^{[\mathbf{n}]} \mathbf{t}^{\mathbf{n}}=\frac{M}{N} \sum_{[\mathbf{n}]=0}\left(\frac{r}{R}\right)^{[\mathbf{n}]} \\
& =\frac{M}{N} \sum_{\mu=0}^{\infty}\left(\frac{r}{R}\right)^{\mu}=M / N\left(1-\frac{r}{R}\right), \quad R>r,
\end{aligned}
$$

that is, the power series in (18) will be absolutely convergent. We thus have the following theorem.

Theorem 1. If the radius of convergence of the series in (23) is equal to $N R$, then series (18) will be absolutely convergent for all matrices situated in the neighborhood of domain (21).

\section{Mode of Increase of FSCMVs}

In this section, we prove two main results which provide the mode of increase of the entire FSCMVs. These results then enable us to compute the growth order and the growth type of some entire FSCMVs.

Let $\mathscr{F}(\mathbf{X})$ be an entire function of several complex matrix variables of common order $N$ with Taylor expansion:

$$
\mathscr{F}(\mathbf{X})=\sum_{[\mathbf{n}]=0}^{\infty} a_{\mathbf{n}} \mathbf{X}^{\mathbf{n}},
$$

and the maximum modulus

$$
M\left[\mathscr{F} ; \overline{\mathbf{S}}_{R}\right]=\max _{i, j} \max _{\left|X_{1}\right|<\left\|r t_{1}\right\| \ldots\left|X_{k}\right|<\left\|r t_{k}\right\|}|\mathscr{F}(\mathbf{X})| .
$$

Therefore, Cauchy's inequality for the matrix function $\mathscr{F}(\mathbf{X})$ can be given in the form 


$$
\left|a_{\mathbf{n}}\right| \leq \frac{N M\left[\mathscr{F} ; \overline{\mathbf{S}}_{r}\right]}{(r N)^{[\mathbf{n}]}} \sigma_{\mathbf{n}} .
$$

For the description of the asymptotic growth behavior of the maximum modulus of entire matrix functions, we write the following.

Definition 1. Let $\mathscr{F}(\mathbf{X})$ be an entire function of several complex matrices. Then, the order of growth of the maximum modulus of an entire function of several complex matrices is described by

$$
\rho(\mathscr{F})=\limsup _{r \longrightarrow \infty} \frac{\ln \ln M\left[\mathscr{F} ; \overline{\mathbf{S}}_{r}\right]}{\ln r} .
$$

Now, we are going to prove our main result which provides us with a generalization to the context of entire FSCMV s of the famous theorem of Lindelöf and Pringsheim in terms of the relation between the growth order and the Taylor coefficients.

Theorem 2. For an entire function in several complex matrix variables with a Taylor series representation of form (28), let

$$
\Omega=\limsup _{[\mathbf{n}] \longrightarrow \infty} \frac{[\mathbf{n}] \ln ([\mathbf{n}])}{-\ln \left|a_{\mathbf{n}} / K_{\mathbf{n}}\right|}, \quad K_{\mathbf{n}}=\frac{\sigma_{\mathbf{n}}}{N^{[\mathbf{n}]} .}
$$

Then, $\rho(\mathscr{F})=\Omega$.

Proof. We first show that $\rho(\mathscr{F}) \geq \Omega$. For $\Omega=0$, this inequality is trivial. So, let us assume that $0<\Omega \leq+\infty$ in what follows. In view of (32), there exist infinitely many $\mathbf{n} \in \mathbb{N}_{0}^{k}$ with

$$
[\mathbf{n}] \ln ([\mathbf{n}]) \geq-b \ln \left|\frac{a_{\mathbf{n}}}{K_{\mathbf{n}}}\right|,
$$

where $b$ is a real constant to be chosen such that $b=\Omega-$ $\varepsilon>0$ with an $\varepsilon>0$ if $\Omega<\infty$. In the case where $\Omega=\infty$, one can take for $b$ any arbitrary positive real number. We then have

$$
\ln \left|\frac{a_{\mathbf{n}}}{K_{\mathbf{n}}}\right| \geq-\frac{[\mathbf{n}]}{b} \ln ([\mathbf{n}]) .
$$

The coefficients of a matrix Taylor series (28) satisfy Cauchy's inequality in the form

$$
\left|a_{\mathbf{n}}\right| \leq N K_{\mathbf{n}} r^{-[\mathbf{n}]} M\left[\mathscr{F} ; \overline{\mathbf{S}}_{r}\right] .
$$

Thus, we may infer that

$$
\begin{gathered}
\ln M\left[\mathscr{F} ; \overline{\mathbf{S}}_{r}\right] \geq \ln \left(\left|\frac{a_{\mathbf{n}}}{K_{\mathbf{n}}}\right| \frac{r^{[\mathbf{n}]}}{N}\right) \\
=\ln \left(\left|\frac{a_{\mathbf{n}}}{K_{\mathbf{n}}}\right|\right)+[\mathbf{n}] \ln \frac{r}{N^{1 /[\mathbf{n}]} .}
\end{gathered}
$$

From relation (33), we find that

$$
\ln M\left[\mathscr{F} ; \overline{\mathbf{S}}_{r}\right] \geq[\mathbf{n}] \ln \frac{r}{N^{1 /[\mathbf{n}]}}-\frac{[\mathbf{n}]}{b} \ln ([\mathbf{n}]) .
$$

Now, let $r=(e[\mathbf{n}])^{1 / b}$ in (37), and we get for this arbitrarily large $r$ the following inequality:

$$
\begin{array}{r}
\ln M\left[\mathscr{F} ; \overline{\mathbf{S}}_{r}\right] \geq[\mathbf{n}] \ln \frac{(e[\mathbf{n}])^{1 / b}}{N^{1 /[\mathbf{n}]}}-\frac{[\mathbf{n}]}{b} \ln ([\mathbf{n}]) \\
=\frac{[\mathbf{n}]}{b}\left(1-\ln N^{b /[\mathbf{n}]}\right)=\frac{r^{b}}{b e}\left(1-\ln N^{b e / r^{b}}\right) .
\end{array}
$$

This permits us to conclude that

$$
\rho(\mathscr{F})=\limsup _{r \longrightarrow \infty} \frac{\ln \ln M\left[\mathscr{F} ; \overline{\mathbf{S}}_{r}\right]}{\ln r} \geq b=\Omega-\varepsilon,
$$

for arbitrary $\varepsilon>0$. For $\varepsilon \longrightarrow 0$, one gets $\rho(\mathscr{F}) \geq \Omega$.

For the case $\Omega \geq \rho(\mathscr{F})$ we see the following.

In the case where $\Omega=+\infty$, there is nothing to prove. So, let us assume without loss of generality that $0 \leq \Omega<\infty$. Since $\mathscr{F}(\mathbf{X})$ is an entire matrix function, we have that $\lim _{[\mathbf{n}] \longrightarrow \infty}\left|a_{\mathbf{n}}\right|=0$. Because of this property and in view of (32), one can find that, for all $\varepsilon>0, \kappa \in \mathbb{N}$ such that for all multi-indices $[\mathbf{n}]$ with $[\mathbf{n}] \geq \kappa$,

$$
0 \leq \frac{[\mathbf{n}] \ln ([\mathbf{n}])}{-\ln \left|a_{\mathbf{n}} / K_{\mathbf{n}}\right|} \leq \Omega+\varepsilon .
$$

For all these multi-indices with $[\mathbf{n}] \geq \kappa$, it holds that

$$
\left|\frac{a_{\mathbf{n}}}{K_{\mathbf{n}}}\right| \leq[\mathbf{n}]^{-[\mathbf{n}] / \Omega+\varepsilon} .
$$

Therefore,

$$
\begin{aligned}
M\left[\mathscr{F} ; \overline{\mathbf{S}}_{r}\right] & \leq \max _{i, j} \max _{\overline{\mathbf{S}}_{r}} \sum_{[\mathbf{n}]=0}\left|a_{\mathbf{n}}\right|\left|\mathbf{X}^{\mathbf{n}}\right| \\
& \leq \frac{1}{N} \sum_{[\mathbf{n}]=0}^{\infty}(N r) \frac{[\mathbf{n}]}{\sigma_{\mathbf{n}}},
\end{aligned}
$$

that is,

$$
\begin{aligned}
M\left[\mathscr{F} ; \overline{\mathbf{S}}_{r}\right] & \leq \frac{1}{N}\left\{\sum_{[\mathbf{n}]=0}^{\kappa}+\sum_{[\mathbf{n}]=\kappa+1}^{\infty}\right\}(N r)^{[\mathbf{n}]} \frac{\left|a_{\mathbf{n}}\right|}{\sigma_{\mathbf{n}}} \\
& \leq \frac{1}{N}\left\{\mathscr{L}_{1}+\sum_{[\mathbf{n}]=\kappa+1}^{\infty}\left(\frac{r^{\Omega+\varepsilon}}{[\mathbf{n}]}\right)^{[\mathbf{n}] / \Omega+\varepsilon}\right\},
\end{aligned}
$$

where $\mathscr{L}_{1}$ is a positive real constant. Choose the number $r_{0}>1$ such that

$$
\left(2 r_{0}\right)^{\Omega+\varepsilon}>\mu \text { for } r>r_{0}, \quad \mu \in \mathbb{N},
$$

and then fix the positive integer $\varepsilon$ such that

$$
\begin{aligned}
\kappa<\varepsilon \leq & \left(2 r_{0}\right)^{\Omega+\varepsilon}<\varepsilon+1 ; \quad r>r_{0}, \\
M\left[\mathscr{F} ; \overline{\mathbf{S}}_{r}\right] \leq & \frac{1}{N}\left\{\mathscr{L}_{1}+\sum_{[\mathbf{n}]=\kappa+1}^{\varepsilon}\left(\frac{r^{\Omega+\varepsilon}}{[\mathbf{n}]}\right)^{[\mathbf{n}] / \Omega+\varepsilon}\right. \\
& \left.+\sum_{[\mathbf{n}]=\varepsilon+1}^{\infty}\left(\frac{r^{\Omega+\varepsilon}}{[\mathbf{n}]}\right)^{[\mathbf{n}] / \Omega+\varepsilon}\right\} .
\end{aligned}
$$


Thus,

$$
\begin{aligned}
\sum_{[\mathbf{n}]=\kappa+1}^{\varepsilon}\left(\frac{r^{\Omega+\varepsilon}}{[\mathbf{n}]}\right)^{[\mathbf{n}] / \Omega+\varepsilon} & <r^{\varepsilon} \sum_{[\mathbf{n}]=\kappa}^{\varepsilon} \frac{1}{(\kappa+1)^{[\mathbf{n}] / \Omega+\varepsilon}} \\
< & r^{\varepsilon} \sum_{[\mathbf{n}]=0}^{\infty} \frac{1}{(\kappa+1)^{[\mathbf{n}] / \Omega+\varepsilon}}<r^{\left(2 r_{0}\right)^{\Omega+\varepsilon}} \\
& \cdot\left\{1-\frac{1}{(\kappa+1)^{1 / \Omega+\varepsilon}}\right\}^{-2}=\mathscr{L}_{2} r^{\left(2 r_{0}\right)^{\Omega+\varepsilon}}, \\
\sum_{[\mathbf{n}]=\varepsilon+1}^{\infty}\left(\frac{r^{\Omega+\varepsilon}}{[\mathbf{n}]}\right)^{[\mathbf{n}] / \Omega+\varepsilon} & \leq \sum_{[\mathbf{n}]=\varepsilon+1}^{\infty}\left(\frac{r^{\Omega+\varepsilon}}{\varepsilon+1}\right)^{[\mathbf{n}] / \Omega+\varepsilon} \\
< & \sum_{[\mathbf{n}]=0}^{\infty}\left(\frac{1}{2}\right)^{[\mathbf{n}] / \Omega+\varepsilon}=\mathscr{L}_{3} .
\end{aligned}
$$

Summarizing, we have obtained

$$
M\left[\mathscr{F} ; \overline{\mathbf{S}}_{r}\right] \leq \mathscr{L}_{4} \exp \left\{(2 r)^{\Omega+\varepsilon} \ln r\right\},
$$

where $\mathscr{L}_{2}, \mathscr{L}_{3}$, and $\mathscr{L}_{4}$ are constants. Making $r \longrightarrow \infty$ such that $\rho(\mathscr{F}) \leq \Omega+\varepsilon$ for $\varepsilon$ tends to zero, and then we arrive at the desired estimate $\rho(\mathscr{F}) \leq \Omega$, and the theorem is hereby deduced.

To get a finer classification of the growth behavior within the set of entire matrix functions that have the same growth order, one further introduces the growth type of an entire matrix functions as follows.

Definition 2. For any entire function of several complex matrices $\mathscr{F}(\mathbf{X})$ of order $\rho(0<\rho<\infty)$, the growth type $\tau$ is given by

$$
\tau=\limsup _{r \rightarrow \infty} \frac{\ln M\left[\mathscr{F} ; \overline{\mathbf{S}}_{r}\right]}{r^{\rho}} .
$$

The following is the second main result which provides us with a generalization of the famous Pringsheim-Lindelöf result on the relation between the growth type and the Taylor coefficients (see, e.g., $[1,2]$ ) in the framework of matrix function theory.

Theorem 3. If $\mathscr{F}(\mathbf{X})$ is an entire matrix function of finite growth order $\rho(0<\rho<\infty)$ and growth type $\tau$, then

$$
\tau=\frac{N^{\rho}}{e \rho} \limsup _{[\mathbf{n}] \longrightarrow \infty}([\mathbf{n}])\left\{\frac{\left|a_{\mathbf{n}}\right|}{\sigma_{\mathbf{n}}}\right\}^{\rho /[\mathbf{n}]} .
$$

To prove this theorem, we need the following lemma.
Lemma 1. Let $\mathscr{F}(\mathbf{X})$ be a function in several complex matrix variables of common order $N$ which have a Taylor series expansion in (28). Suppose there are numbers $\alpha>0$ and $\beta>0$ and an integer $\gamma=\gamma(\alpha, \beta)>0$ such that $\left(\left|a_{\mathbf{n}}\right| / \sigma_{\mathbf{n}}\right)<$ $\left(e \alpha \beta / N^{\alpha}[\mathbf{n}]\right)^{[\mathbf{n}] / \alpha}$ for all $[\mathbf{n}]>\gamma$. Then, $\mathscr{F}(\mathbf{X})$ is an entire matrix function, and given any $\varepsilon>0$, there is a number $R=$ $R(\varepsilon)>0$ such that

$$
M\left[\mathscr{F} ; \overline{\mathbf{S}}_{r}\right]<\exp \left\{(\beta+\varepsilon) r^{\alpha}\right\} \text { for all } r>R .
$$

Proof. Since $\left(\left|a_{\mathbf{n}}\right| / \sigma_{\mathbf{n}}\right)<\left(e \alpha \beta / N^{\alpha}[\mathbf{n}]\right)^{[\mathbf{n}] / \alpha}$, for all these multi-indices with $[\mathbf{n}]>\gamma$, it holds that

$$
\left(\frac{N^{[\mathbf{n}]}\left|a_{\mathbf{n}}\right|}{\sigma_{\mathbf{n}}}\right)^{1 /[\mathbf{n}]}<\left(\frac{e \alpha \beta}{[\mathbf{n}]}\right)^{1 / \alpha}
$$

Hence, $\left(N^{[\mathbf{n}]}\left|a_{\mathbf{n}}\right| / \sigma_{\mathbf{n}}\right)^{1 /[\mathbf{n}]} \longrightarrow 0,[\mathbf{n}] \longrightarrow \infty$, and $\mathscr{F}(\mathbf{X})$ is an entire matrix function.Furthermore,

$$
\left(\frac{(N r)^{[\mathbf{n}]}\left|a_{\mathbf{n}}\right|}{\sigma_{\mathbf{n}}}\right)^{1 /[\mathbf{n}]}<\left(\frac{e \alpha \beta}{[\mathbf{n}]}\right)^{1 / \alpha} r<1 / 2,
$$

if multi-indices with $[\mathbf{n}]>\kappa=\kappa(r)=\left(2^{\alpha} e \alpha \beta r^{\alpha}\right)$. Choosing $R_{1}=R_{1}(\alpha, \beta)>1$, which is so large that $\kappa(r)>\gamma$, and if $r>R_{1}$, then

$$
\left(\frac{(N r)^{[\mathbf{n}]}\left|a_{\mathbf{n}}\right|}{\sigma_{\mathbf{n}}}\right)<1 / 2^{[\mathbf{n}]},
$$

provided multi-indices with $[\mathbf{n}]>\kappa$.

We now establish an upper bound for $M\left[\mathscr{F} ; \overline{\mathbf{S}}_{r}\right]$ :

$$
\begin{aligned}
M\left[\mathscr{F} ; \overline{\mathbf{S}}_{r}\right] & \leq \max _{i, j} \max _{\overline{\mathbf{S}}_{r}} \sum_{[\mathbf{n}]=0}^{\infty}\left|a_{\mathbf{n}}\right|\left|\mathbf{X}^{\mathbf{n}}\right| \\
& \leq \frac{1}{N} \sum_{[\mathbf{n}]=0}^{\infty} \frac{\left|a_{\mathbf{n}}\right|}{\sigma_{\mathbf{n}}}(N r)^{[\mathbf{n}]} \\
& =\frac{1}{N} \sum_{[\mathbf{n}]=0}^{\kappa} \frac{\left|a_{\mathbf{n}}\right|}{\sigma_{\mathbf{n}}}(N r)^{[\mathbf{n}]}+\frac{1}{N} \sum_{[\mathbf{n}]=\kappa+1}^{\infty} \frac{\left|a_{\mathbf{n}}\right|}{\sigma_{\mathbf{n}}}(N r)^{[\mathbf{n}]} \\
& <\sum_{[\mathbf{n}]=0}^{\kappa} \frac{\left|a_{\mathbf{n}}\right|}{\sigma_{\mathbf{n}}}(N r)^{[\mathbf{n}]}+\sum_{[\mathbf{n}]=\kappa+1}^{\infty} \frac{1}{2^{[\mathbf{n}]}} \\
& <\sum_{[\mathbf{n}]=0}^{\kappa} \frac{\left|a_{\mathbf{n}}\right|}{\sigma_{\mathbf{n}}}(N r)^{[\mathbf{n}]}+1, \quad \text { if } r>R_{1} .
\end{aligned}
$$


However,

$$
\begin{aligned}
& \sum_{[\mathbf{n}]=0}^{\kappa} \frac{\left|a_{\mathbf{n}}\right|}{\sigma_{\mathbf{n}}}(N r)^{[\mathbf{n}]}= \sum_{[\mathbf{n}]=0}^{\gamma} \frac{\left|a_{\mathbf{n}}\right|}{\sigma_{\mathbf{n}}}(N r)^{[\mathbf{n}]}+\sum_{[\mathbf{n}]=\gamma+1}^{\kappa} \frac{\left|a_{\mathbf{n}}\right|}{\sigma_{\mathbf{n}}}(N r)^{[\mathbf{n}]} \\
&<r^{\gamma} \sum_{[\mathbf{n}]=0}^{\gamma} \frac{\left|a_{\mathbf{n}}\right|}{\sigma_{\mathbf{n}}} N^{[\mathbf{n}]}+(\kappa-\gamma) \\
& \\
& \max _{\gamma+1 \leq[\mathbf{n}] \leq \kappa} \frac{\left|a_{\mathbf{n}}\right|}{\sigma_{\mathbf{n}}}(N r)^{[\mathbf{n}]} \leq \max _{\gamma+1 \leq[\mathbf{n}] \leq \kappa} \frac{\left|a_{\mathbf{n}}\right|}{\sigma_{\mathbf{n}} \mid}(N r)^{[\mathbf{n}]}, \\
& \sigma_{\mathbf{n}}(N r)^{[\mathbf{n}]} \\
&<\max _{\gamma+1 \leq[\mathbf{n}]}\left(\frac{e \alpha \beta}{N^{\alpha}[\mathbf{n}]}\right)^{[\mathbf{n}] / \alpha}(N r)^{[\mathbf{n}]} \\
& \leq \max _{1 \leq[\mathbf{n}]}\left(\frac{e \alpha \beta}{[\mathbf{n}]}\right)^{[\mathbf{n}] / \alpha} r^{[\mathbf{n}]}=\exp \left\{\beta r^{\alpha}\right\} .
\end{aligned}
$$

The maximum is achieved for all these multi-indices with $[\mathbf{n}]=\beta \alpha r^{\alpha}$; hence,

$$
\max _{\gamma+1 \leq[\mathbf{n}] \leq \kappa} \frac{\left|a_{\mathbf{n}}\right|}{\sigma_{\mathbf{n}}}(N r)^{[\mathbf{n}]}<\exp \left\{\beta r^{\alpha}\right\} .
$$

Therefore, if $r>R_{1}$,

$$
\begin{aligned}
M\left[\mathscr{F} ; \overline{\mathbf{S}}_{r}\right]< & r^{\gamma} \sum_{[\mathbf{n}]=0}^{\gamma} \frac{\left|a_{\mathbf{n}}\right|}{\sigma_{\mathbf{n}}} N^{[\mathbf{n}]}+(\kappa-\gamma) \exp \left\{\beta r^{\alpha}\right\}+1 \\
= & r^{\gamma} \sum_{[\mathbf{n}]=0}^{\gamma} \frac{\left|a_{\mathbf{n}}\right|}{\sigma_{\mathbf{n}}} N^{[\mathbf{n}]}+\left(2^{\alpha} e \alpha \beta r^{\alpha}-\gamma\right) \exp \left\{\beta r^{\alpha}\right\}+1 \\
= & \exp \left\{\beta r^{\alpha}\right\}\left\{2^{\alpha} e \alpha \beta r^{\alpha}-\gamma+\exp \left\{-\beta r^{\alpha}\right\}\right. \\
& \left.+r^{\gamma} \exp \left\{-\beta r^{\alpha}\right\} \sum_{[\mathbf{n}]=0}^{\gamma} \frac{\left|a_{\mathbf{n}}\right|}{\sigma_{\mathbf{n}}} N^{[\mathbf{n}]}\right\} .
\end{aligned}
$$

Given any $\varepsilon>0$, there is a number $R=R(\varepsilon)>R_{1}$ such that the expression in brackets is less than $\exp \left\{\varepsilon r^{\alpha}\right\}$ provided that $r>R$. Hence,

$$
M\left[\mathscr{F} ; \overline{\mathbf{S}}_{r}\right]<\exp \left\{(\beta+\epsilon) r^{\alpha}\right\} \text { for all } r>R .
$$

Now Lemma 1 can be used to determine Theorem 3 as follows.

Proof of Theorem 3.2. Suppose $\tau$ is finite. Then, given any $\pi>\tau$, there is a number $R=R(\pi)>0$ such that $M\left[\mathscr{F} ; \overline{\mathbf{S}}_{r}\right]<\exp \left\{\pi r^{\rho}\right\}$, for $r>R$. According to Cauchy's inequality in (30), we have

$$
\begin{aligned}
N^{[\mathbf{n}]}\left|\frac{a_{\mathbf{n}}}{\sigma_{\mathbf{n}}}\right| & \leq \frac{N M\left[\mathscr{F} ; \overline{\mathbf{S}}_{r}\right]}{r^{[\mathbf{n}]}} \\
& <\frac{N \exp \left\{\pi r^{\rho}\right\}}{r^{[\mathbf{n}]}}, \text { for all } r>R .
\end{aligned}
$$

The minimum value of $\exp \left\{\pi r^{\rho}\right\} / r^{[\mathbf{n}]}$ occurs for $r=([\mathbf{n}] / \pi \rho)^{1 / \rho} ;$ thus,

$$
N^{[\mathbf{n}]}\left|\frac{a_{\mathbf{n}}}{\sigma_{\mathbf{n}}}\right|<N\left(\frac{e \rho \pi}{[\mathbf{n}]}\right)^{[\mathbf{n}] / \rho},
$$

for all these multi-indices with $[\mathbf{n}]>\gamma$ and $r=([\mathbf{n}] / \pi \rho)^{1 / \rho}>R(\pi)$.

Rewriting,

$$
N^{\rho /[\mathbf{n}]} \pi>\frac{N^{\rho}}{e \rho}([\mathbf{n}])\left\{\left|\frac{a_{\mathbf{n}}}{\sigma_{\mathbf{n}}}\right|\right\}^{\rho /[\mathbf{n}]} .
$$

Hence,

$$
\pi \geq \frac{N^{\rho}}{e \rho} \limsup _{[\mathbf{n}] \longrightarrow \infty}([\mathbf{n}])\left\{\left|\frac{a_{\mathbf{n}}}{\sigma_{\mathbf{n}}}\right|\right\}^{\rho /[\mathbf{n}]} .
$$

Since $\pi$ is an arbitrary number exceeding $\tau$,

$$
\tau \geq \frac{N^{\rho}}{e \rho} \limsup _{[\mathbf{n} \sqsupset \longrightarrow \infty}([\mathbf{n}])\left\{\left|\frac{a_{\mathbf{n}}}{\sigma_{\mathbf{n}}}\right|\right\}^{\rho /[\mathbf{n}]},
$$

where the right-hand side is clearly finite. Now let $\pi_{1}$ be any number exceeding the right-hand side of (49). Then, there is a number $\gamma=\gamma\left(\pi_{1}\right)>0$ such that

$$
N^{[\mathbf{n}]}\left|\frac{a_{\mathbf{n}}}{\sigma_{\mathbf{n}}}\right|<\left(\frac{e \rho \pi_{1}}{[\mathbf{n}]}\right)^{[\mathbf{n}] / \rho}, \quad \text { for all }[\mathbf{n}]>\gamma .
$$

Applying Lemma 1 with $\beta=\pi_{1}$ and $\alpha=\rho$, given such that

$$
M\left[\mathscr{F} ; \overline{\mathbf{S}}_{r}\right]<\exp \left\{\left(\pi_{1}+\epsilon\right) r^{\rho}\right\}, \quad \text { for all } r>R .
$$

Therefore, $\tau \leq \pi_{1}$ and because of the choice of $\pi_{1}$,

$$
\tau \leq \frac{N^{\rho}}{e \rho} \limsup _{[\mathbf{n}] \longrightarrow \infty}([\mathbf{n}])\left\{\left|\frac{\mid a_{\mathbf{n}}}{\sigma_{\mathbf{n}}}\right|\right\}^{\rho /[\mathbf{n}]} .
$$

Thus, the result is derived. Also, if the right-hand side of (49) is finite so is $\tau$, and if $\tau$ is infinite, so is the right-hand side of (49).

3.1. Basic Applications. Theorem 2 and Theorem 3 allow us to evaluate the exact value of the growth order and the growth type for some elementary matrix generalizations of the classical exponential, trigonometric, and other special functions. To illustrate this, let us afford here some important examples.

Example 1. Let

$$
\mathscr{F}(\mathbf{X}):=e^{\sum_{s=1}^{k} b_{s} X_{s}}, \quad s=1,2, \ldots, k,
$$


be an entire function of several complex matrix variables $\mathbf{X}=\left(X_{1}, X_{2}, \ldots, X_{k}\right)$ all of which are of common order $N$ in $\overline{\mathbf{S}}_{r} ; b_{s}$ are positive numbers, and then the growth order $\rho(\mathscr{F})=1$ and the growth type $\tau=k \sqrt{b_{1}^{2}+b_{2}^{2}+\cdots+b_{k}^{2}}$.

Proof. Definition 1 and Definition 2 tell us that

$$
\begin{gathered}
\rho=\limsup _{r \rightarrow \infty} \frac{\ln \ln M\left[\mathscr{F} ; \overline{\mathbf{S}}_{r}\right]}{\ln r}, \\
\tau=\limsup _{r \rightarrow \infty} \frac{\ln M\left[\mathscr{F} ; \overline{\mathbf{S}}_{r}\right]}{r^{\rho}} .
\end{gathered}
$$

Thus,

$$
\begin{array}{r}
|\mathscr{F}(\mathbf{X})|=\left|e^{\sum_{s=1}^{k} b_{s} X_{s}}\right|, \quad s=1,2, \ldots, k, \\
M\left[\mathscr{F} ; \overline{\mathbf{S}}_{r}\right]=\max _{i, j} \max _{\mathbf{X} \in \overline{\mathbf{S}}_{r}}|\mathscr{F}(\mathbf{X})| \\
=\max _{i, j} \max _{\mathbf{X} \in \overline{\mathbf{S}}_{r}} e^{\left|\sum_{s=1}^{k} b_{s} X_{s}\right|}=e^{k r \sqrt{b_{1}^{2}+b_{2}^{2}+\cdots+b_{k}^{2}}} .
\end{array}
$$

This leads to

$$
\begin{array}{r}
\rho=\limsup _{r \rightarrow \infty} \frac{\ln \left(k r \sqrt{b_{1}^{2}+b_{2}^{2}+\cdots+b_{k}^{2}}\right)}{\ln r}=1, \\
\tau=\limsup _{r \rightarrow \infty} \frac{\left(k r \sqrt{b_{1}^{2}+b_{2}^{2}+\cdots+b_{k}^{2}}\right)}{r}=k \sqrt{b_{1}^{2}+b_{2}^{2}+\cdots+b_{k}^{2}} .
\end{array}
$$

Remark 1. One could expect to get entire function of several complex matrices of growth order $p(p \in \mathbb{N})$ and growth type $\left(k \sqrt{b_{1}^{2}+b_{2}^{2}+\cdots+b_{k}^{2}}\right)^{p}$ just by taking

$$
\mathscr{F}(\mathbf{X})=e^{\left(\sum_{s=1}^{k} b_{s} X_{s}\right)^{p}}, \quad s=1,2, \ldots, k .
$$

Example 2. The generalized cosine function of several complex matrices $\mathbf{X}=\left(X_{1}, X_{2}, \ldots, X_{k}\right)$, all of which are of common order $N$ in $\overline{\mathbf{S}}_{r}$, given by

$$
\cos (\mathbf{X}):=\sum_{[\mathbf{n}]=0}^{\infty} \frac{(-1)^{[\mathbf{n}]}}{(2 \mathbf{n}) !} X_{1}^{2 n_{1}}, X_{2}^{2 n_{2}}, \ldots, X_{k}^{2 n_{k}},
$$

where $2 \mathbf{n}=\left(2 n_{1}, 2 n_{2}, \ldots, 2 n_{k}\right)$ have growth order $\rho=1$ and growth type $\tau=N k$.

Proof. First, we note that the power series in (72) has indeed an infinite radius of convergence in $\overline{\mathbf{S}}_{r}$; thus, the essential

$$
\frac{1}{N} \sum_{[2 \mathbf{n}]=0}^{\infty} \frac{1}{(2 \mathbf{n}) !} \cdot \frac{(N r)^{[2 \mathbf{n}]}}{\sigma_{2 \mathbf{n}}}
$$

converges for all $0 \leq r<\infty$ in $\overline{\mathbf{S}}_{r}$.

Applying Theorem 2 and Theorem 3 lead immediately to

$$
\begin{aligned}
\rho & =\limsup _{[\mathbf{n}] \longrightarrow \infty} \frac{2[\mathbf{n}] \ln (2[\mathbf{n}])}{\ln \left|\sigma_{2 \mathbf{n}} /\left((-1)^{[\mathbf{n}]} /(2 \mathbf{n}) !\right) N^{2[\mathbf{n}]}\right|} \\
& =\limsup _{[\mathbf{n}] \longrightarrow \infty} \frac{2[\mathbf{n}] \ln (2[\mathbf{n}])}{\ln \sigma_{2 \mathbf{n}}+\ln (2 \mathbf{n}) !-2[\mathbf{n}] \ln N} .
\end{aligned}
$$

According to Stirling's formula in the form

$$
[\mathbf{n}] ! \sim[\mathbf{n}]^{[\mathbf{n}]+(1 / 2)} \sqrt{2 \pi} e^{-[\mathbf{n}]},
$$

(see [13] and [14]) we get $\rho=1$, and if [n] is sufficiently large we have

$$
\left[\left(\frac{2[\mathbf{n}]}{k}\right) !\right]^{k} \leq(2 \mathbf{n}) ! \leq(2[\mathbf{n}]) !
$$

This leads to

$$
\begin{aligned}
\tau & =\frac{N^{\rho}}{e \rho} \limsup _{[\mathbf{n}] \longrightarrow \infty}(2[\mathbf{n}])\left\{\left|\frac{(-1)^{[\mathbf{n}]} /(2 \mathbf{n}) !}{\sigma_{2 \mathbf{n}}}\right|\right\}^{\rho / 2[\mathbf{n}]} \\
& =\frac{N}{e} \limsup _{[\mathbf{n}] \longrightarrow \infty}(2[\mathbf{n}])\left\{\left|\frac{1 /(2 \mathbf{n}) !}{\sigma_{2 \mathbf{n}}}\right|\right\}^{1 / 2[\mathbf{n}]} \\
& =\frac{N}{e} \limsup _{[\mathbf{n}] \longrightarrow \infty} e^{\ln (2[\mathbf{n}])-(1 /|2[\mathbf{n}]|)\left(\ln \mid(2 \mathbf{n}) !+\ln \sigma_{2 \mathbf{n}}\right)} \\
& =\frac{N}{e} \limsup _{[\mathbf{n}] \longrightarrow \infty} e^{\ln (2[\mathbf{n}])-(1 /|2[\mathbf{n}]|)\left(k \ln (2[\mathbf{n}] / k) !+\ln \sigma_{2 \mathbf{n}}\right)}=N k
\end{aligned}
$$

Example 3. Similarly, one can show that the generalized sine function of several complex matrices $\mathbf{X}=\left(X_{1}, X_{2}, \ldots, X_{k}\right)$ all of which are of common order $N$ in $\overline{\mathbf{S}}_{r}$ by

$$
\sin (X)=\sum_{[\mathbf{n}]=0}^{\infty} \frac{(-1)^{[\mathbf{n}]}}{(2 \mathbf{n}+1) !} X_{1}^{2 n_{1}+1}, X_{2}^{2 n_{2}+1}, \ldots, X_{k}^{2 n_{k}+1}
$$

where $2 \mathbf{n}+1=\left(2 n_{1}+1,2 n_{2}+1, \ldots, 2 n_{k}+1\right)$ also have growth order $\rho=1$ and growth type $\tau=N k$.

Example 4. We can use the same construction principle to get examples of the rational growth order and growth type. This elementary example is, for instance, the generalized Bessel matrix function of order 0 of several complex matrices $\mathbf{X}=\left(X_{1}, X_{2}, \ldots, X_{k}\right)$ all of which are of common order $N$ in $\overline{\mathbf{S}}_{r}$ defined by

$$
J_{0}(\mathbf{X}):=\sum_{[\mathbf{n}]=0}^{\infty} \frac{1}{(\mathbf{n} !)^{2}} X_{1}^{n_{1}}, X_{2}^{n_{2}}, \ldots, X_{k}^{n_{k}}
$$

and then using Theorem 2 and Theorem 3, we have its growth order $\rho=(1 / 2)$ and growth type $\tau=0$.

Example 5. According to Theorem 2 and Theorem 3. All matrix power series 


$$
\mathscr{P}(\mathbf{X}):=\sum_{[\mathbf{n}]=0}^{N} a_{\mathbf{n}} \mathbf{X}^{\mathbf{n}}
$$

have growth order $\rho=0$ and growth type $\tau=\infty$.

\section{Linear Substitution for FSCMs}

Let $\mathscr{F}^{\star}(\mathbf{X})=\mathscr{F}(\mathbf{X}+\mathbf{A})$ be an entire matrix function of growth order $\rho^{\star}$ and growth type $\tau^{\star}$, where $\mathbf{A}=\left[a_{s: i j}\right], s=$ $1,2, \ldots, k, i, j=1,2, \ldots, N$ are any constant numbers. It is required in this section to establish $\rho^{\star}$ and $\tau^{\star}$ as follows.

If $\mathbf{X}=\left(\left[x_{s ; i j}\right]\right), s=1,2, \ldots, k$, are several complex matrices situated in the neighbourhood of the origin given by (21), then

$$
\begin{aligned}
\sum_{s=1}^{k}\left|x_{s ; i j}+a_{s ; i j}\right| & \leq \sup _{|\mathbf{t}|=1}\left(r^{k}+2 r\left(t_{1}+t_{2}+\cdots+t_{k}\right)+\cdots+k \theta^{k}\right) \\
& =(r+\sqrt{k} \theta)^{k}=R^{k} ; \\
\theta & =\sup _{i, j}\left(\left|a_{1 ; i j}\right|,\left|a_{2 ; i j}\right|, \ldots,\left|a_{k ; i j}\right|\right) .
\end{aligned}
$$

The matrices $\mathbf{X}+\mathbf{A}$ are situated in the neighbourhood of the origin given by

$$
|\mathbf{X}+\mathbf{A}|<\|R \mathbf{t}\|, \quad|\mathbf{t}|=1, R=r+\sqrt{k} \theta .
$$

Thus,

$$
M\left[\mathscr{F}^{\star} ; \overline{\mathbf{S}}_{r}\right] \leq M\left[\mathscr{F} ; \overline{\mathbf{S}}_{r}\right]
$$

Now, suppose that $\varepsilon>0$ is any number, then there is a positive number $r_{1}$ such that, applying (48) and (83), one gets

$$
M\left[\mathscr{F}^{\star} ; \overline{\mathbf{S}}_{r}\right] \leq M\left[\mathscr{F} ; \overline{\mathbf{S}}_{r}\right]<e^{\varepsilon R^{\rho}}, \quad \text { for } R>r_{1} .
$$

That is,

$$
\begin{aligned}
\rho^{\star} & =\limsup _{r \longrightarrow \infty} \frac{\ln \ln M\left[\mathscr{F}^{\star} ; \overline{\mathbf{S}}_{r}\right]}{\ln r} \\
& \leq \limsup _{r \rightarrow \infty} \frac{\ln \epsilon(r+\sqrt{k} \theta)^{\rho}}{\ln r}=\rho .
\end{aligned}
$$

On the other hand, if $\mathscr{F}^{\star}(\mathbf{X}-\mathbf{A})=\mathscr{F}(\mathbf{X})$, then $\rho \leq \rho^{\star}$, and thus $\rho=\rho^{\star}$.

Considering the growth type $\tau^{\star}$, we see from (84) and (48) that

$$
\begin{aligned}
\tau^{\star} & =\limsup _{r \longrightarrow \infty} \frac{\ln M\left[\mathscr{F}^{\star} ; \overline{\mathbf{S}}_{r}\right]}{r^{\rho^{*}}} \\
& \leq \limsup _{r \rightarrow \infty} \frac{\epsilon(r+\sqrt{k} \theta)^{\rho}}{r^{\rho}}=\epsilon .
\end{aligned}
$$

Since $\epsilon$ can be chosen arbitrarily as near to $\tau$ as we please, we infer that $\tau^{\star} \leq \tau$. In the usual way, if $\mathscr{F}^{\star}(\mathbf{X}-\mathbf{A})=\mathscr{F}(\mathbf{X})$, we infer that $\tau^{\star} \geq \tau$, and hence $\tau^{\star}=\tau$. Thus, the following theorem is determined.
Theorem 4. The entire matrix function $\mathscr{F}^{\star}(\mathbf{X})$ is of the same growth order and growth type as the matrix function $\mathscr{F}(\mathbf{X})$.

Now, we consider the matrix function $\mathscr{F}^{\star}(b \mathbf{X}+\mathbf{A}), b \neq 0$, and $\mathscr{F}(\mathbf{X})$ are an entire matrix function of growth order $\rho$ and growth type $\tau$. Then, we obtained the following result by modicum calculations based on Theorem 4 and relations (31) and (48).

Theorem 5. Let $\mathscr{F}(\mathbf{X})$ be an entire matrix function of positive order $\rho$ and type $\tau$, and let $b \neq 0$ be the real constant. Then, the matrix function $\mathscr{F}^{\star}(b \mathbf{X}+\mathbf{A})$ is an entire matrix function of the growth order $\rho$ and the growth type $\tau|b|^{\rho}$ [26].

\section{Data Availability}

No data were used to support this study.

\section{Conflicts of Interest}

The authors declare that they have no conflicts of interest.

\section{Authors' Contributions}

All the authors contributed equally and significantly to writing this article. All the authors read and approved the final manuscript.

\section{Acknowledgments}

The authors extend their appreciation to the Deanship of Scientific Research at King Khalid University for funding this work through the Research Groups Program under Grant no. R.G.P.1/153/40.

\section{References}

[1] E. Lindelöf, "Sur la détermination de la croissance des fonctions entiéres définies par un développement de Taylor," Darb. Bulletin, vol. 27, pp. 213-226, 1903.

[2] A. Pringsheim, "Elementare Theorie der ganzen transzendenten Funktionen von endlicher Ordnung," Mathematische Annalen, vol. 58, no. 3, pp. 257-342, 1904.

[3] G. Valiron, Lectures on the General Theory of Integral Functions, Chelsea, New York, NY, USA, 1949.

[4] S. M. Shah, "On the lower order of integral functions," Bulletin of the American Mathematical Society, vol. 52, no. 12, pp. 1046-1052, 1946.

[5] S. M. Shah, "On the coefficients of an entire series of finite order," Journal of the London Mathematical Society, vol. 26, no. 1, pp. 45-46, 1951.

[6] A. A. Gol'dberg, "Elementary remarks on the formulas defining the order and type entire functions in several variables," Doklady Akademiya Nauk Armyanskoi SSR, vol. 29, pp. 145-151, 1959.

[7] R. K. Dutta, "On order of a function of several complex variables analytic in the unit polydisc," Kragujevac Journal of Mathematics, vol. 36, pp. 163-174, 2012.

[8] L. I. Ronkin, Introduction to the theory of entire functions of several variables, Translations of Mathematical Monographs, vol. 44, American Mathematical Society, Providence, RI, USA, 
[9] R. K. Srivastava and V. Kumar, "On the order and type of integral functions of several complex variables," Compositio Mathematica, vol. 17, pp. 161-166, 1966.

[10] T. Winiarski, "Application of approximation and interpolation methods to the examination of entire functions of $n$ complex variables," Annales Polonici Mathematici, vol. 28, no. 1, pp. 97-121, 1973.

[11] M. A. Abul-Ez and R. De Almeida, "On the lower order and type of entire axially monogenic functions," Results in Mathematics, vol. 63, no. 3-4, pp. 1257-1275, 2013.

[12] R. Brualdi and D. Cvetkvić, A Combinatorial Approach to Matrix Theory and its Applications, Chapman and Hall/CRC, New York, NY, USA, 2009.

[13] D. Constales, R. De Almeida, and R. S. Krausshar, "On the relation between the growth and the Taylor coefficients of entire solutions to the higher dimensional Cauchy-Riemann system in $R^{n+1}$," Journal of Mathematical Analysis and Applications, vol. 327, no. 2, pp. 763-775, 2007.

[14] D. Constales, R. De Almeida, and R. S. Krausshar, "On the growth type of entire monogenic functions," Archiv der Mathematik, vol. 88, no. 2, pp. 153-163, 2007.

[15] D. Constales, R. De Almeida, and R. S. Krausshar, "Applications of the maximum term and the central index in the asymptotic growth analysis of entire solutions to higher dimensional polynomial Cauchy-Riemann equations, Complex Var," Complex Variables and Elliptic Equations, vol. 53, no. 3, pp. 195-213, 2008.

[16] G. S. Srivastava and S. Kumar, "On the generalized order and generalized type of entire monogenic functions," Demonstratio Mathematica, vol. 46, no. 4, pp. 663-677, 2013.

[17] N. J. Higham, Functions of Matrices Theory and Computation, SIAM, University City, MI, SA, 2008.

[18] Z. Kishka, M. Abul-Ez, M. Saleem, and H. Abd-Elmaged, "On the order and type of entire matrix functions in complete Reinhardt domain," Journal of Modern Methods in Numerical Mathematics, vol. 3, pp. 31-40, 2012.

[19] Z. Kishka, "Functions of several square commutative complex matrices," Bulletin of Science Quarterly Journal Pure and Applied Mathematics, vol. 66, pp. 69-77, 1992.

[20] V. L. A. Smirnov, Course of Higher Mathematics, Pergamon Press, vol. III2, Oxford, UK, 4th edition, 1964.

[21] M. Nassif, "Composite sets of polynomials of several complex variables," Publicationes Mathematicae Debrecen, vol. 18, pp. 43-52, 1971.

[22] K. A. Sayyed and M. S. Metwally, “ $(p, q)$-order and $(p, q)$-type of basic sets of polynomials of two complex variables," Complex Variables, vol. 26, pp. 63-68, 1994.

[23] M. Gil, Localization and Perturbation of Zeros of Entire Functions, Taylor and Francis Group, LLC, New York, NY, USA, 2010.

[24] B. Ya. Levin, Lectures on Entire Functions, American Mathematical Society, Providence, RI, USA, 1996.

[25] M. N. Seremeta, "On the connection between the growth of the maximum modulus of an entire function and the moduli of the coefficients of its power series expansion," American Mathematical Society Translations, vol. 88, pp. 291-301, 1970.

[26] R. De Almeida and R. S. Krausshar, "Basics on growth orders of polymonogenic functions, Complex Var," Complex Variables and Elliptic Equations, vol. 60, no. 11, pp. 1-25, 2015. 